\title{
Optimal Use Of ANN In The Integration Between Digital Image Processing And Encryption Technique
}

\author{
Shaimaa Abbas Fahdel Al-Abaidy \\ University of Baghdad / College of Education for Pure Science Ibn Al Haitham
}

Article History: Received:11 January 2021; Accepted: 27 February 2021; Published online: 5 April 2021

\begin{abstract}
Recently An ANN is the very much powerful and strong technology for solving various real world-real time issues in area of image processing such as medical, research center etc. This paper represents the Artificial Neural network in combination with the image processing. The main aim of this paper is to study the ANN in integration with digital image processing and encryption technologies. So in this sense we discussed the basics of image processing, digital image processing, artificial neural network and encryption. With the help of proposed block diagram we discussed ANN with and without encryption. When an ANN is used with digital image processing several algorithms are available. Here we discussed the few algorithms in shortly with their iteration equation used at time of learning the ANN.
\end{abstract}

Keywords: Digital image processing, pixel, Encryption, Artificial Neural Network, optimization algorithm

\section{Introduction:}

Today different multimedia's are utilized by everyone such as android cellphones, personal digital assistant (PDAs).These are wireless system allow to store and exchange of data. Advance technologies are ready to handle all videos as well as digital images strongly. So Digital image processing is the important aspect of digital signal processing. When any information is transferred through the network not only it is must to maintain privacy and security for that data but also recover that data with quality. So the technique is available for keeping privacy and security named as encryption. To fulfill the requirement of channel encryption is preferable. Encryption is needed to hide the data in image. Encryption supports to minimize the attacks from the third party. Similarly Artificial intelligence is also plays an important role in digital signal processing [1].

ANN is the computation system whose inspiration is biological neural network present in human brain. Artificial neuronal networks in combination with Image processing is successfully utilized in different fields such as civil engineering, geo-technics, image recognition, speech recognition, medical field industrial surveillance, mechanics, automation, defense department and transport systems. Here this topic deal with use of artificial neural network along with digital image processing and image encryption technique which provides better result than when they work individually. Management of preprocessing of image, data reduction, and segmentation data (image) and recognition of data are the main steps carried out for management of image with the help of ANN [2] and [3].

Organization of this paper is as: In Section 2 discusses the Basic concepts such as image processing, Digital image processing, Artificial Neural Network along with encryption technique. In Section 3 includes Integration between digital image processing with ANN and Integration between image encryption with ANN. Section IV, includes basic block diagram of ANN with working and some mathematical derivations. It also includes optimal use of ANN using various algorithms used for training of neural network. Section V and section VI summarizes the conclusion and references respectively.

\section{Basic Concepts:}

Under this section, basics terms related to this topic are discussed.

\subsection{Image processing:}

Image processing becomes necessary feature to retrieve maximum important information acceptable for different applications such as Material Science, Graphic arts, Medical Imaging, Remote Sensing, Forensic Studies, Non-destructive Evaluation, Textiles, Film industry, Military, Printing Industry and Document processing[4]. Basic steps carried out in image processing are scanning of image, image enhancement, Interpretation and hoard the image. Enhancing the quality of image can be possible traditional as well as modern techniques. Image processing is popularly employed in various fields to achieve the quality output. Degradation of image is one of the serious issue occur in wireless transmission. Generally, Digital image processing is a solution for challenges, issues such that loss 
of quality of image and enhancement of degraded image. It is deal with image analysis, extraction of feature and restores an image.

There are the two different types of processing of image i.e. analog image processing and digital image processing. In case of analog image processing there is amendment of image using the electrical variation technique. Television image is the Best example for analog image processing. Voltage level indicates the television signal. Variation in voltage level causes the change in amplitude which makes the change in brightness of the image. Hence electrical signal variation changes the image appearance which altered. Various knobs on television set allow as changing in appearance of image with respect to reference signal [5].

\subsection{Digital Image processing:}

Digital image processing is most important aspect in variety of application area. The processing of image is startups with news industry because of computer technology and with very high speed it is dominant in many professions. Generally image processing is the techniques which enhance the output of system whose input is the raw or worst quality of image captured by the camera or sensor placed on space probe, aircraft and satellites [6].

Digital image processing allows the use of computer with various techniques along with numerical representation or two dimensional processing which results into desired result. It gets input as a raw image and produces image with quality appearance. Digital image is the two dimensional representation of image having set of finite digital values. These finite values are known as pixel i.e. picture element .It represents intersection of row and column. Pixels are related with brightness value or digital number. Generally Digital no ranges from 0-63 i.e. b bit data, 0-255 i.e. 8 bit data and 0-1063 i.e. 10 bit data. This represents average radiance of respective tiny area within the scene [1].

Consider image be abstract (Mathematically continuous, discrete and two variable), non- perceptible (It is related to field parameters like pressure, density, temperature) or perceptible (seen because of the light intensity).On the basis of data of image means matrix, the image is divided into scalar image and vector image. In scalar image each element is unique having real number pixel value while in vector image each element is spitted into many parts. Image is represented as matrix, $M=m \times n$, each array component contains with information of the colour of pixel [7].

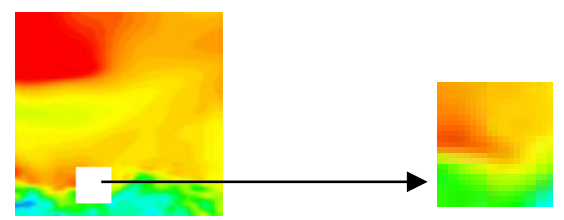

Colour image Pixel with colour information

Fig 1:Pixel

\subsection{Artificial Neural Network and Model of ANN:}

Now days, Artificial neural network becomes very popular and useful model in various cases such as clustering, prediction, classification and pattern recognition. It is top most model of machine learning. It becomes adequately aggressive to ordinary regression and the statistical model concerning usefulness [8].

Artificial Intelligence includes the concept neural network, machine learning, and deep learning. Recently cloud computing, Artificial intelligence, information security, internet, forensic techniques (science) are hotspots inspiring scenario for information \& communication technology. Artificial neural network are used in many applications with reference to analysis of various factors like scalability, accuracy, tolerance, processing speed [9] and [10].

ANN plays an important role in field of digital image processing. There are various techniques are used for error free or less error output with integration of ANN such as image encryption, image decryption etc. Below Figure 1 shows the basic structure of ANN. It is 3 layer structures: Input layer, Hidden layer and output layer. Here hidden layer consist of many stages. 


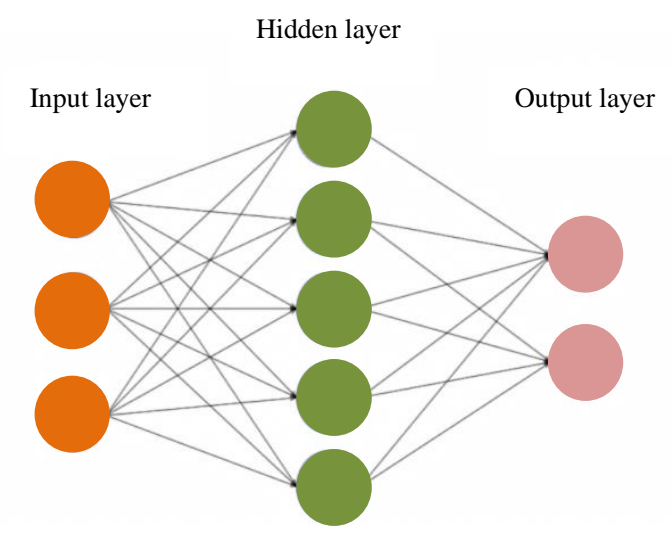

Basically it is an interconnection of group of nodes which is inspired by simple structure of neuron in human brain. In above fig circular node stands for artificial neuron. Each arrow represents the connection between this neuron in the sense ones output is input for next stage neuron. Neurons, Connections with respective weights and Propagation function are the main components of the ANN [11].

In fig 2 represents the components of ANN. w is the weight present at connection. I indicate the input while $\mathrm{O}$ indicates the output. $\mathrm{x}$ represents the set of inputs while $\mathrm{F}$ is the output

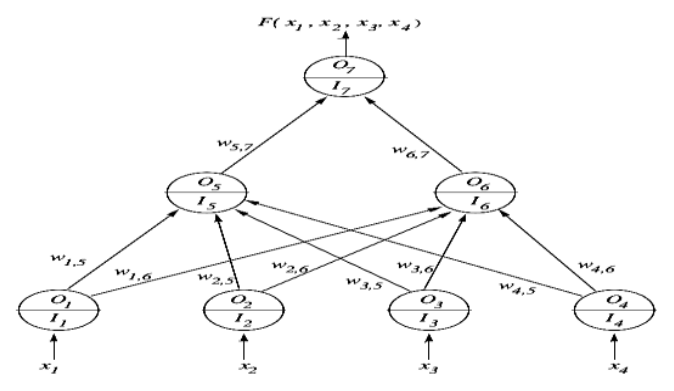

Fig 3: Components of ANN

of that network which is function of input $\mathrm{x}$. The main aim of the ANN is to solve the complex problem same as human brain. Hence special feature of ANN may allow using it in digital image processing on conjunction with encryption to get the quality output [12].

The mathematical representation of above network is as follows,

At input side,

$$
\text { Node input: } \text { net }_{l}=\sum_{l=1}^{4} w_{i, j} x_{l}
$$

At output side,

$$
\text { Node output: } O_{l}=f\left(\text { net }_{l}\right)
$$

\subsection{Encryption of Image:}

Encryption technique furnishes the security for important data. Place to place the data is varying when it is transmitted through the wireless medium. But if data transmits after the encryption process then it becomes secured. As information is in the encrypted form then it becomes difficult to hacker to access it. Hence highly privacy is maintained. Personal important data and issues are kept secured because of encryption process [13]. 


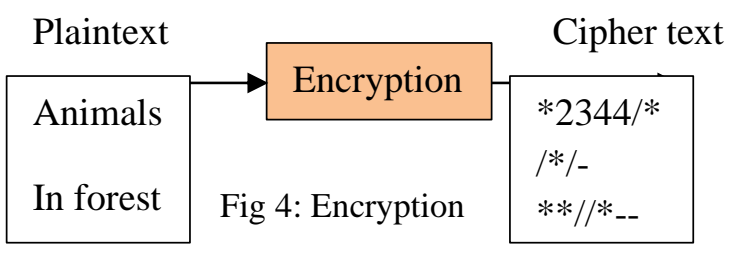

A perfect Encryption provides security to data. General steps carried out in encryption are: the confidential and important data is converted it the cipher text which is random manner. Then acquired cipher is scattered over the network. Recovered cipher at receiver sider is then processed by applying decryption algorithm to get the plain text. Effective and Enhanced security can be obtained with combination of spatial domain encryption technique and traditional stream cipher [14].

\section{Integration between Digital Image processing and encryption technique with ANN:}

Now a days ANN and digital image processing are working together to achieve the quality output. Here we discuss the combination of digital image processing and encryption technique with ANN on the basis of computation time as well as quality of output result.

\subsection{Integration between Digital Image processing with ANN:}

ANN is computational model which is inspired by learning ability of a human intelligence nerve system. Basically it is structure processing method. Special characteristics of ANN make it is prominent. It has more number of weighted connections between those distributed elements. An ANN is highly utilized since it has enormous Parallelism nature, Distributed rendition with learning ability and fault tolerance. Main module of ANN are processing unit, topologies and learning algorithms.

A digital image is represented in the form of matrix. Each value of matrix represents colour information of a pixel. This matrix is using as input for the neural network. Image is divided into many small parts. Hence small dimensions or values of image can quickly and easily help learning of network, set up the vector size and input vector numbers [15].

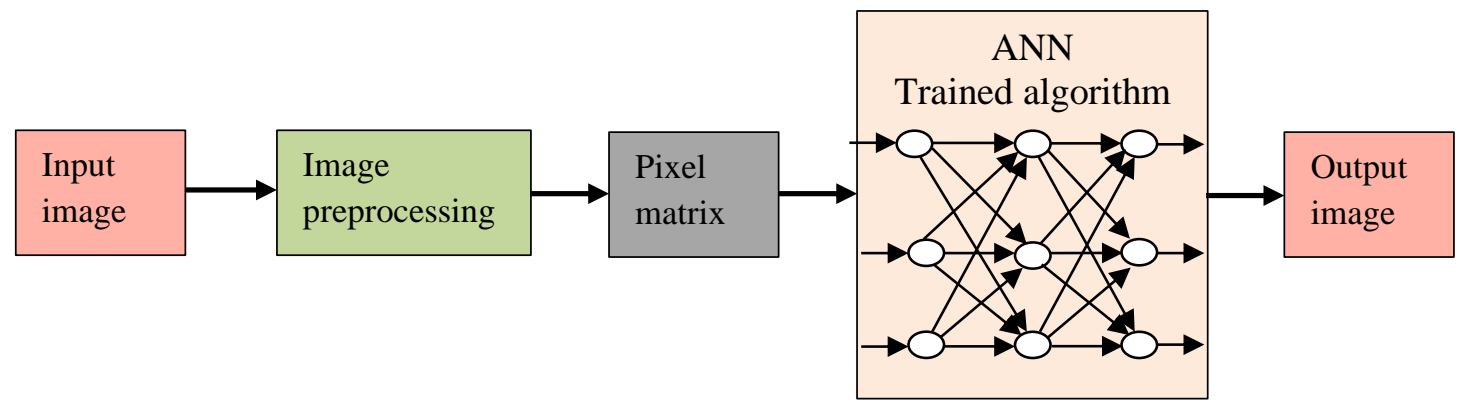

Fig 5: Basic block diagram of Digital image processing is in integration with ANN

Fig shows the basic block diagram of the ANN system without encryption. Here just the segment or piecewise image is processed with ANN and then transmitted. The output quality of these system is poor as compare to ANN with encryption since the transmitted image will not protected from the channel attack .so anyone can easily access the data and modify it and hence we will not receive actual quality output.

\subsection{Integration between image encryption with ANN:}

As per previous discussion, image encryption plays an important role if a security is important issue. In bellows fig the encryption algorithm is applied to the output of neural network block. Hence whatever the data is processed by ANN is encrypted and then it is transmitted over the wireless medium. In presence of encryption technique, it is difficult to access the information by third party (Hackers) and due to ANN as it works on array of matrix simultaneously on basis of input defined by user, it requires less computational time. Reduction in the computational time by increasing computational speed is main advantages of ANN [15]. 


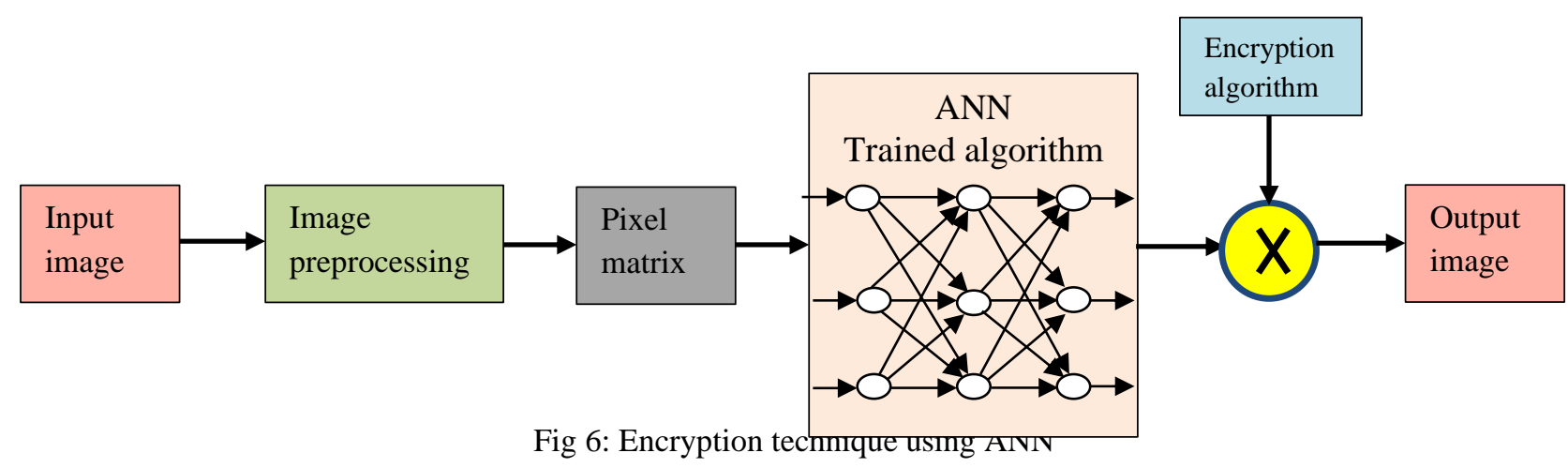

\section{Basic block diagram of ANN with working and some mathematical derivations}

Cryptography is one of the important scenarios if we deal with encryption and decryption. Hence two things are come in picture: chaotic cryptosystems and ANN based chaotic system. Below fig shows the complete architecture of the ANN based system. The signal generated by control unit is controls chaotic sequence generator where direct encryption of an image is obtained. It support to high security to multi system. Generated sequence is then given to ANN block for training the sequence [14] and [16].

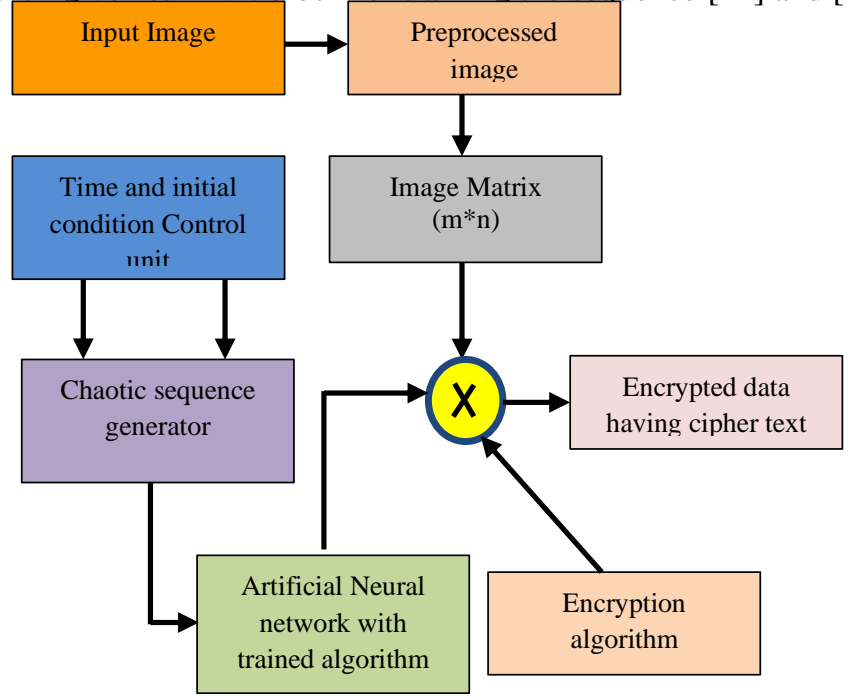

Transmitter

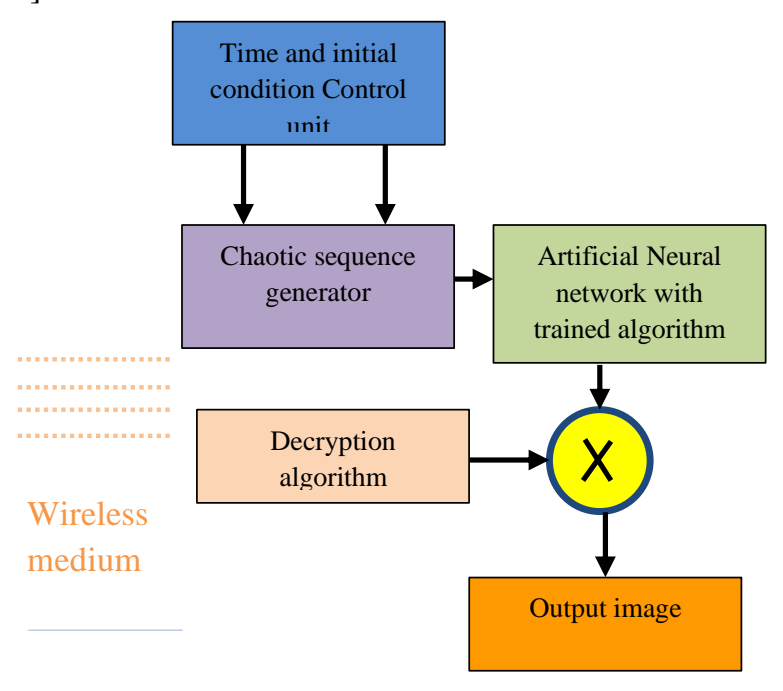

Receiver

Fig 7: Architecture of ANN Based Digital signal processing system

With the help of encryption algorithm the image is encrypted with the cipher. After the encryption the image is transmitted over the wireless medium. At receiver side the received image is decrypted.

\subsection{Optimal use of ANN:}

Highly complex data is easily processed by ANN. Adaptive learning means the ability of learning how to complete task based on given initial conditions or training. Self-organization is more specific characteristics of ANN. An ANN generates its self or own organization (representation) of information which is received at time of learning time. An ANN can works on the real time applications. All ANN computations are carried parallel manner using the specific hardware which can easily utilizes the all capabilities of ANN [17].

ANN supports too many features and more advantages than the traditional processing method or techniques. During the learning the activation function determines the next stage of activation based on effective input. Effective input is determined by propagation rule. If there is presence of error in input then also system prates 
in this environment. Back propagation algorithm is one of the most important algorithms which are responsible to reduce the error. Single layer feed forward neural network have many restrictions. Back-propagation is best solution for those types of errors. The hidden layer error is determined by back propagating errors of output layer. This is known as back propagation learning rule. It can be termed as generalization of the delta rule in case of multilayer function [3] and [18].

Generalized delta rule is

$$
\delta_{h}^{p}=\mathcal{F}^{\prime}\left(s_{h}^{p}\right) \sum_{i=0}^{n} \delta_{i}^{p} w_{h i} \quad i=, 1,2 \ldots \ldots \ldots n
$$

\subsection{Role of ANN in image processing:}

\subsubsection{Image reconstruction}

Reconstruction of image on the basis parameter obtained from sensor is termed as image reconstruction. Reconstruction problem recommends complex computation. Each application needs unique approach. In case Hopfield network is used to reconstruct the computerized image (tomography images) which performs inverse radon transform. This network consist of 'Adding ' layer'. Regression feed-forward network is used by Meyer and Heindl [19] for reconstructing the image from electron hologram. Hopfield ANN was trained by Wang and Wahl to reconstruction the 2D image on pixel data getting from projection [20] and [21].

\subsubsection{Image restoration}

Removal of aberrations occurred due to sensor and noise from an image is called as Image restoration. Basically it is restoration of image from distorted data from measurement system. Motion blur, noise, out-of-focus blur caused distortion and hence we found less resolution. Various designs are available in combination with ANN to restore the image.one important approach is filtering. Greenhil and Davies was used regression feed-forward network for suppressing in convolution [22]. Guan et al. develop system for reconstruction of image in which each module is separate ANN [23].

\subsubsection{Image enhancement}

Amplification of few features from image is termed as image enhancement.Eage detection is an most important scenario in image enhancement. regression feed-forward ANN is trained in such that it behaves like edge detector was explained by Pugmire et al [24]. ART network was used by Shih et al. to enhance the binary image [25].

\subsubsection{Image compression:}

Self-organizing Maps (SOMs), feed-forward networks, adaptive fuzzy leader clustering are various ANN trained to do image compression. Parameter adaptation is the Main advantages of ANN which gave better rate of compression with specific image data using traditional compression method [26].

\subsubsection{Feature extraction applications}

Basically it is data reduction techniques .It is helpful to find Subset of specific feature which anyone wants. Eg. Face recognition used for security purpose. Unsupervised and supervised ANNs are used to extract he feature. These methods are more advantageous than traditional method [27]

\subsubsection{Image segmentation:}

Many ANN techniques were reported by researchers in which segmentation of images directly get through pixel data. Number of ANNs were present to do pixel-based segmentation such as SOMs, feed-forward ANNs, probabilistic ANNs, Hopfield networks, CNNs, , constraint satisfaction and radial basis function networks ANNs. Generally on basis of pixel supervised ANN was trained to distinguish image on texture, edge pixels connections, identify the surfaces and pixel clustering [28].

\section{Optimization}

The procedure utilized to perform learning or training algorithm in artificial neural network is known as optimization algorithm. It is also called as optimizer. Gradient descent, Newton's method, Conjugate gradient, Quasi-Newton method and Levenberg-Marquardt algorithm are few important algorithms used to train the network [29].

5.1.1 The iteration equation for gradient decent method

$$
w^{(j+1)}=w^{j}-g^{j} \eta^{j} \quad \text { for } \quad j=, 1,2 \ldots \ldots \ldots n
$$




\section{Optimal Use Of ANN In The Integration Between Digital Image Processing And Encryption}

\section{Technique}

Here $\eta$ is training rate and its value may be fixed. Its value can be finding out by $1 \mathrm{D}$ optimization in each step of training. Training rate optimal value is obtained via line minimization which highly preferable.

5.1.2 Newton's method is generally iterated by following equation.

$$
\begin{gathered}
w^{(j+1)}=w^{j}-\left(H^{j-1} g^{j}\right) \eta \quad \text { for } \quad j=, 1,2 \ldots \ldots \ldots n \\
\text { where } g=g^{0}+H^{0}\left(w-w^{0}\right)=0 \quad \text { for } \quad j=, 1,2 \ldots \ldots \ldots n
\end{gathered}
$$

Newton's method is difficult for exact evaluation for Hessian as well as its inverse. It is highly expensive as computational point of view.

4.1.3 Conjugate gradient algorithm can be iterated by using the equation

$$
w^{(j+1)}=w^{j}+d^{j} \eta^{j} \quad \text { for } \quad j=, 1,2 \ldots \ldots \ldots n
$$

Where

$$
d^{(j+1)}=g^{j+1}+d^{j} \gamma^{j} \quad \text { for } \quad j=, 1,2 \ldots \ldots \ldots n
$$

4.1.4 Quasi-Newton method uses the following equation of iteration

$$
w^{(j+1)}=w^{j}-\left(G^{j} g^{j}\right) \eta^{j} \quad \text { for } \quad j=, 1,2 \ldots \ldots \ldots n
$$

4.1.5 Levenberg-Marquardt algorithm is also termed as least square algorithm. It is generally working with loss function.

Loss function is mathematically represented as

$$
\begin{array}{cc}
f=\sum_{i=1}^{n} e_{i}^{2} & m=\text { instance no in data set } \\
J_{i, K}=\frac{\delta e_{i}}{\delta w_{k}} & i=1, \ldots, n \text { anf } j=1, \ldots n
\end{array}
$$

So this method is iterated by following equation

$$
w^{(j+1)}=w^{j}-\left(\left(J^{j T} J^{j}\right)+\lambda^{j}\right)^{-1} \cdot\left(2 J^{j T} \cdot e^{j}\right) \text { for } \quad j=, 1,2 \ldots \ldots \ldots
$$

Damping parameter $\lambda$ is zero [30],[31] and [32].

To implementation of all those algorithms are possible with MATLAB. It is high level computational language. It links between domain for computation, programming and visualization. MATLAB provides an Image Processing Toolbox. It has various references set with standard algorithms as well as functions. Also includes different apps which are useful for image processing. The toolbox is very easy to handle. It works with high speed .Also it becomes convenient way for processing, analyzing an images with simple coding language.

\section{Conclusion}

This paper provides the basics of image processing along with artificial neural network. Artificial Neural Networks becomes powerful technique which is able to reproduce highly complex computational machines. We can be used this technique for building simple and easy sequential machine. Implementation of Artificial Neural Networks is possible with combinational and sequential circuits. The use of ANN in the field of image processing in combination with encryption provides high security to confidential data. Hence ANN is responsible to solve 
complex issues having high speed and les computational time. Various algorithms are available to train the network which provides improved result. To reduce error in single layer network back-propagation algorithm will preferable. So ANN will use as new approach for encryption and decryption of image for getting quality output.

\section{References:}

[1] Muzamil Bhat (2014). Digital Image Processing. International Journal Of Scientific \& Technology Research ,3(1),pp.272-276.

[2] Jure Zupan (1984). Introduction to Artificial Neural Network (ANN) Methods: What They Are and How to Use Them. Acta Chimica Slovenica 41/3/1994, research gate, pp. 327-352.

[3] Mina Niknafs . Neural Network Optimization.pp1-7.

[4] Ravindra S. Hegadi (2010). Image Processing: Research Opportunities and Challenges. National Seminar on Research in Computers,pp.1-5.

[5] S. Muthuselvi and P. Prabhu(2016). Digital Image Processing Techniques - A Survey, International Multidisciplinary Research Journal,5(11),pp.1-11

[6] Dr. Uddin N.U.,Dr. Jebunnahar and Md. Bashar A. (2012). A Comprehensive Study of Digital Image Processing for Finding Image Quality Dependencies. International Journal of Scientific and Research Publications,2(3),pp.1-8.

[7] Alexandrina-Elena P.,, Mihai B.And Gabriela C. (2015). Image Processing Using Artificial Neural Networks. Bul. Inst. Polit. Iaşi, t. LXI (LXV),pp.9-21.

[8] V.S. Dave, K. Dutta (2014).Neural network-based models for software effort estimation: a review, Artif. Intell. Rev., 42 (2) , pp. 295-307.

[9] H. He, E.A. Garcia (2009).Learning from imbalanced data. IEEE Trans. Knowl. Data Eng., 21 (9) ,pp. 1263-1284.

[10] A. Mozaffari, M. Emami, A. Fathi (2018).A comprehensive investigation into the performance, robustness, scalability and convergence of chaos-enhanced evolutionary algorithms with boundary constraints Artif. Intell. Rev. , pp. 1-62

[11] Ghosh A. and Dr. Nath A. (2014) Cryptography Algorithms using Artificial Neural Network. International Journal of Advance Research in Computer Science and Management Studies,2(11),pp.375-381.

[12] Ms.Dharmistha and D.Vishwakarma(2012). Genetic Algorithm based Weights Optimization of Artificial Neural Network . International Journal of Advanced Research in Electrical, Electronics and Instrumentation Engineering,1(3),pp.206-211.

[13] Priya Deshmukh(2016).An image encryption and decryption using AES algorithm, IJSER ,7(2).pp.210213.

[14] Minal Chauhan and Rashmin Prajapati (2014).Image Encryption Using Chaotic Based Artificial Neural Network. International Journal of Scientific \& Engineering Research,5(6) , pp.351-354.

[15] Tope K., Rane A., Rahate R. and Asst. Prof. Nalawade S.M.(2015). Encryption and Decryption using Artificial Neural Network. International Advanced Research Journal in Science, Engineering and Technology,2(4),pp.81-83.

[16] Quist-Aphetsi Kester(2013) .A Cryptographic Image Encryption Technique For Facial-Blurring Of Images , IJATER, 3(3).pp.

[17] Iain M. C.,Rebecca H. and Stern S.(2018). The Impact Of Artificial Intelligence On Innovation. National Bureau Of Economic Research,pp.1-40.

[18] Hong Hui Tan and King Hann Lim (2019). Review of second-order optimization techniques in artificial neural networks backpropagation. IOP Conf. Series: Materials Science and Engineering, 495,pp.1-8.

[19] R.R. Meyer, E. Heindl (1998). Reconstruction of off axis electron holograms using a neural net, Journal of Microscopy, 191 (1) pp. 52-59.

[20] Y.M. Wang, F.M. Wahl (1997). Vector-entropy optimization-based neural-network approach to image reconstruction from projections, IEEE Transactions on Neural Networks ,8 (5), 1008-1014.

[21] Rajesh G, Dr.A.Muthukumaravel (2016). Role of Artificial Neural Networks (ANN) in Image Processing. International Journal of Innovative Research in Computerand Communication Engineering,4(8), pp.14509-14516.

[22] D. Greenhil and E.R. Davies(1994). Relative effectiveness of neural networks for image noise suppression, Proc. Pattern Recognition in Practice IV, Vlieland, pp. 367-378. 
[23] L. Guan, J.A. Anderson, J.P. Sutton (1997). A network of networks processing model for image regularization, IEEE Transactions on Neural Networks 8 (1), pp.169-174.

[24] R.H. Pugmire, R.M. Hodgson, R.I. Chaplin(1998). The properties and training of a neural network based universal window filter developed for image processing tasks, in: Brain-like computing and intelligent information systems, S. Amari and N. Kasabov, eds., Springer-Verlag, Singapore, pp. 49-77.

[25] F.Y. Shih, J. Moh, F.-C. Chang (1992). A new ART based neural architecture for pattern classification and image enhancement without prior knowledge, Pattern Recognition ,25 (5), pp.533542 .

[26] C. Amerijckx, M. Verleysen andP. Thissen (1998). Image compression by self-organized Kohonen map, IEEE Transactions on Neural Networks, 9 (3), pp.503-507.

[27] A. Ghosh(1995) Use of fuzziness measures in layered networks for object extraction: a generalization, Fuzzy Sets and Systems 72 (3), pp.331-348

[28] M.N. Ahmed and A.A. Farag (1997). Two-stage neural network for volume segmentation of medical images. Pattern Recognition Letters 18, (11-13), pp.11431151.

[29] Shiliang Sun, Zehui Cao, Han Zhu, and Jing Zhao(2019). A Survey of Optimization Methods from a Machine Learning Perspective.pp.1-30.

[30] Rossana M. S. Cruz 1 , Helton M. Peixoto 2 and Rafael M. Magalhães. Artificial Neural Networks and Efficient Optimization Techniques for Applications in Engineering,pp.46-68

[31] G. Venter. Review of Optimization Techniques. Research Gate, Encyclopedia Of Aerospace Engineering. pp.1-13.

[32] Harrison MonFook Leong. Optimiza On With Artificial Neural Network Systems: A Mapping Principle And A Comparison To Gradient Based Methods ,pp.474-484 